\title{
Sequential Residual Generator Selection for Fault Detection
}

\author{
Daniel Eriksson $^{1}$ and Christofer Sundström ${ }^{1}$
}

\begin{abstract}
Structural methods in model-based fault diagnosis applications are simple and efficient tools for finding candidates for residual generation. However, the structural methods do not take model uncertainties and information about fault behavior into consideration. This may result in selecting residual generators with bad performance to be included in the diagnosis system. By using the Kullback-Leibler divergence, the performance of different residual generators can be compared to find the best one. With the ability to quantify diagnostic performance, the design of residual generators can be optimized by, for example, combining several residual generators such that the diagnostic performance is maximized. The proposed method for residual generation selection is applied to a water tank system to show that the achieved residual performance is improved compared to only use a structural method.
\end{abstract}

\section{INTRODUCTION}

Designing a diagnosis system to monitor a physical system is a complex engineering task involving many aspects [1]. A model based diagnosis system is based on residuals, and there will most likely be thousands of residual generator candidates to be included in the system. The selection of which of these candidates to include in the diagnosis system is a key issue to achieve good fault detection performance. There are several works considering methods for finding subsets of model equations with analytical redundancy to be used for residual generation, see e.g., [2]. These methods are helpful to find candidates of possible residuals, but it is time consuming to manually evaluate the residual generator candidates and single out the good residual generators.

In [3], that is based on [4], a structural method for finding, generating residuals, and evaluating algebraic and dynamic properties of the residual generators is proposed. This is a help for an engineer in the design process of the diagnosis system to, e.g., avoid algebraic loops. However, no information about the sensitivity to fault and noise in the residuals is considered. If fault detectability performance of the different residuals could be quantified, the best residual with a certain isolability property can automatically be selected.

The performance of a set of diagnostic tests, e.g., residuals and thresholds, is commonly evaluated using fault detectability and isolability which are deterministic properties, see [5], [6], or evaluated using data from a set of fault scenarios, see references in [7]. The performance of a single test quantity is often evaluated using ROC-curves [8], or power functions [9]. However, early in the design process of the residuals, i.e. before choosing a threshold function, these previous methods

\footnotetext{
This work was partially supported by the Swedish Research Council within the Linnaeus Center CADICS.

${ }^{1}$ The authors are with the Vehicular Systems, Dept. of Electrical Engineering, Linköping University, SE-581 83 Linköping, Sweden, \{daner, csu\}aisy.liu.se.
}

are not sufficient to compare the performance of the residual generator candidates. A quantitative measure of diagnosability performance given a residual generator is necessary, which is not dependent on the use of a threshold function.

In [10] and [11], quantitative detectability and isolablity performance are considered based on the Kullback-Leibler divergence. The possibility of using a quantitative measure of fault diagnosability opens up for performance optimization of diagnosis systems, such as sensor placement, linear residual generation, and automatic test selection, see [12].

Here, an automated method for finding and designing residual generators, combined with a quantitative measure of diagnosability performance, is proposed to optimize the selection of residual generators for improved performance. For evaluation of the method, a model of a watertank system is analyzed to find and design residual generators.

The outline is as follows. First, an illustrative example is considered in Section II where optimal residual generation of a triple-redundancy system is analyzed. Then, a method for automatic construction of residual generators is described in Section III and quantitative diagnosability analysis in Section IV. Optimizing residual generators to improve performance is discussed in Section V. Then, a case-study considering a watertank system is performed in Section VI and finally the conclusions are presented in Section VII.

\section{ILLUSTRATIVE EXAMPLE}

As a motivation to the topic of this paper, an example of triple redundancy is used. Triple redundancy is a hardware redundancy approach for fault-tolerant system design which is commonly used in e.g., air planes [13]. The simplest form of triple redundancy is that three sensors, $y_{1}, y_{2}$, and $y_{3}$, measuring the same signal $x$. A fault in a sensor is here modeled by an additive signal $f_{i}$, and the sensor noise is assumed to be additive independent and identically distributed (i.i.d.) Gaussian, which is denoted $\nu_{i} \in \mathcal{N}\left(0, \sigma_{i}\right)$, as

$$
\begin{array}{ll}
e_{1}: & y_{1}=x+\nu_{1}+f_{1} \\
e_{2}: & y_{2}=x+\nu_{2}+f_{2} \\
e_{3}: & y_{3}=x+\nu_{3}+f_{3} .
\end{array}
$$

There is a freedom in the design process of the residual generators to be used in the diagnosis system. Below are all combinations of the linear residual generators that can be designed, given that $\alpha, \beta$, and $\gamma$ are constants, as

$$
r=\alpha y_{1}+\beta y_{2}+\gamma y_{3} .
$$

To achieve $\mathbb{E}[r]=0$, the following condition needs to be fulfilled

$$
0=\alpha+\beta+\gamma
$$




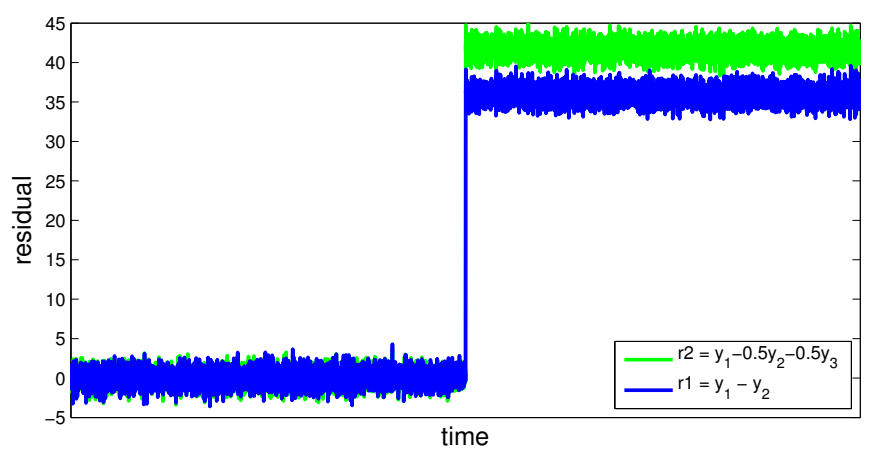

Fig. 1. Example comparing a residual computing the difference of two sensors pair-wise and the residual with maximum fault to noise ratio.

The distribution of $r$ is

$$
r \in \mathcal{N}\left(\alpha f_{1}+\beta f_{2}+\gamma f_{3}, \sqrt{\alpha^{2} \sigma_{1}^{2}+\beta^{2} \sigma_{2}^{2}+\gamma^{2} \sigma_{3}^{2}}\right),
$$

leading to that the fault to noise ratio, $\Gamma$, for $f_{1}$ e.g., is expressed as

$$
\Gamma_{1}=\frac{\alpha f_{1}}{\sqrt{\alpha^{2} \sigma_{1}^{2}+\beta^{2} \sigma_{2}^{2}+\gamma^{2} \sigma_{3}^{2}}} .
$$

It is wanted to maximize the fault to noise ratio to achieve a sensitive diagnosis system. In the example for $f_{1}$ in (4), this leads to the following conditions for $\beta$ and $\gamma$

$$
\begin{aligned}
& \beta=-\frac{\sigma_{3}^{2}}{\sigma_{2}^{2}+\sigma_{3}^{2}} \alpha \\
& \gamma=-\frac{\sigma_{2}^{2}}{\sigma_{2}^{2}+\sigma_{3}^{2}} \alpha .
\end{aligned}
$$

The efficient structural method used to design residual generators, described in e.g. [2], finds minimal sets of equations with redundancy one. Such sets are referred to as a Minimally Structurally Overdetermined (MSO) set of equations, i.e., a set of equations with one more equation than variables and where no proper subset of the equations is structurally overdetermined. Given (1), there are three MSO sets: $\left\{e_{1}, e_{2}\right\},\left\{e_{1}, e_{3}\right\}$, and $\left\{e_{2}, e_{3}\right\}$. Here, it is possible to generate any residual in the form (2a) by taking a linear combination of the three MSOs.

Two residuals to detect $f_{1}$ are compared, one based on the MSO $\left\{e_{1}, e_{2}\right\}, r_{1}=y_{1}-y_{2}$, and one is the linear residual with best fault to noise ratio, given that $f_{1}=50$ and $\sigma_{i}=1$, $i=1,2,3$. The residual with optimal fault to noise ratio for $f_{1}$ is $r_{2}=y_{1}-0.5 y_{2}-0.5 y_{3}$, which gives $\Gamma_{1}=50 \sqrt{\frac{2}{3}}$, but $\Gamma_{1}=50 \frac{1}{\sqrt{2}}$ for $r_{1}$. The two residuals are shown in Fig. 1, and it is visible that the optimized residual $r_{2}$ is more sensitive to $f_{1}$ than $r_{1}$. Thus, the example shows that the detectability performance can be improved by taking model uncertainties and measurement noise into consideration when designing a residual generator.

\section{Automatic Residual Generator COnstruction}

In this section, a method for automatically compute residual generator candidates to be included in a diagnosis system is presented. The inputs to the method are the model equations of the system and data from actuators and sensors in both the fault free case and the faulty cases. The residuals computed are in Sections IV and V evaluated and compared.

Residual generators can be constructed using different methodologies, e.g., parameter estimation [14], and stateobserver [15] techniques. However, here a method based on computation sequences of the unknown variables is used [16], and is called sequential residual generators by [4]. A sequential residual generator consists of a set of equations with analytical redundancy where a subset of the equations are used to solve the unknown variables included in these equations, and then the redundant equations are used to investigate the consistency between the observations and the set of model equations. The basic principle is illustrated with a small example.

Example 1: Consider the model

$$
\begin{aligned}
& e_{1}: x_{1}=g_{1}\left(x_{2}\right) \\
& e_{2}: x_{2}=g_{2}(u) \\
& e_{3}: y=x_{1}
\end{aligned}
$$

with 3 equations, 2 unknown variables $x_{1}$ and $x_{2}$, known input variable $u$, measurement variable $y$, and non-linear functions $g_{i}$. Here, both $e_{1}$ and $e_{3}$ can be chosen as the consistency relation, or analytical redundancy relation (ARR) [17], resulting in the two residual generators

$$
\begin{array}{ll}
x_{2}:=g_{2}(u) & x_{2}:=g_{2}(u) \\
x_{1}:=y & x_{1}:=g_{1}\left(x_{2}\right) \\
r_{1}:=x_{1}-g_{1}\left(x_{2}\right) & r_{2}:=y-x_{1}
\end{array}
$$

respectively.

In the simple example above, the different choices of consistency relations result in equivalent residual generators. However, in general the choice may strongly affect the performance of the diagnosis system, which is shown in Section VI.

The method used to automatically generate the sequential residual generators is described in [3], that is based on [4], but the key ideas are briefly described below.

The first step is to find sets of equations with analytical redundancy one. This is here done by using structural analysis of the model [18]. Structural methods analyze the model structure, i.e. only information about which variables that are included in each equation is used, which is an efficient tool compared to analyzing the algebraic equations.

The set of equations used to construct a residual generator is denoted $\bar{E}$, and it is possible to construct as many sequential residual generators as there are equations in each MSO. This is since every equation in $\bar{E}$ can be selected as the redundant equation $e_{i}$, and the just determined set of equations $\bar{E} \backslash e_{i}$ is used to compute all unknown variables, $X^{\prime}$, included in the specific MSO.

The second step in the method is to find a computation sequence for each residual generator based on the structural analysis, i.e. in which order to compute the unknown variables $x_{i} \in X^{\prime}$ and from which equation each variable is to be 
computed. An algebraic tool is used to rewrite the equations used in the computation sequence to find expressions for the unknown variables. When a nonlinear system is considered, a unique solution for the unknown variables is not guaranteed. Consider e.g. the equation

$$
0=x_{1}-x_{2}^{2}
$$

It is possible to compute a unique value for $x_{1}$ from (6), but there are two solutions for $x_{2}$. Methods for handling multiple solutions are available [3], but in this work only unique residual generators are considered.

Dynamic residual generators often occurs when monitoring physical systems. Integrating a signal is straight forward in the computational sequences, but there are several methods for differentiating a signal, see e.g. [19]. The selection of the differentiation algorithm affects the resulting residuals. A differentiated variable here is computed by comparing two time steps and divide by the time step

$$
\dot{x}_{i}=\frac{x_{i}-x_{i-1}}{\Delta t}
$$

but any other differentiation algorithm can easily be implemented in the computational scheme of the residuals.

Finally, numerical values for the residuals are computed using the known signals, i.e. control signals and measurements.

\section{QUANTITATIVE DIAGNOSABILITY ANALYSIS}

The fault detection performance of a residual generator $r$ is related to how likely an observation of $r$ can be explained by the system being in a faulty state, i.e., $f_{i} \neq 0$, compared to the fault-free state NF (No Fault), i.e., $f_{i}=0, \forall i$. The diagnosability performance of $r$ can be quantified by taking the distributions of $r$ during the faulty and fault-free cases into consideration using the Kullback-Leibler divergence.

\section{A. Kullback-Leibler divergence}

To evaluate and compare the diagnosability performance of different residual generators, a quantitative measure is necessary. The Kullback-Leibler divergence is here used as the quantitative measure, and the benefit of this measure is that performance of the residual generators can be evaluated without considering any specific threshold function [12].

The Kullback-Leibler divergence from a probability density function (pdf) $p$ to another pdf $q$ is defined as

$$
K(p \| q)=\mathrm{E}_{p}\left[\log \frac{p}{q}\right]
$$

and $q \neq 0$ where $p \neq 0$. The Kullback-Leibler divergence is a non-negative, non-symmetric measure, i.e., normally $K(p \| q) \neq K(q \| p)$. Let $p$ be the pdf of $r$ in the faulty case and $q$ the pdf in the fault-free case. Then, (8) can be interpreted as the mean value of the log-likelihood ratio test if $p$ is the true case, i.e., how likely it is to correctly detect a fault. A higher log-likelihood in average represents better detectability performance.
If $p, q \in \mathbb{R}^{k}$ are multivariate Gaussian distributed, $p \sim$ $\mathcal{N}\left(\mu_{p}, \Sigma_{p}\right)$ and $q \sim \mathcal{N}\left(\mu_{q}, \Sigma_{q}\right)$, then (8) can be computed analytically as [9]

$$
\begin{aligned}
K(p \| q) & =\frac{1}{2}\left(\operatorname{tr}\left(\Sigma_{q}^{-1} \Sigma_{p}\right)+\left(\mu_{q}-\mu_{p}\right)^{T} \Sigma_{q}^{-1}\left(\mu_{q}-\mu_{p}\right)\right. \\
& \left.-k-\log \left(\frac{\operatorname{det} \Sigma_{q}}{\operatorname{det} \Sigma_{p}}\right)\right)
\end{aligned}
$$

where $k$ is the dimension of $p$ and $q$.

\section{B. Quantitative residual performance analysis}

By using the Kullback-Leibler divergence, the performance of different residual generators can be compared. Since only the distributions of the residual generators are necessary, and not a threshold which determines if a fault is detected, different solutions of residual generators can be compared early in the design process.

As an example, Fig. 2 shows the pdfs of two residuals sensitive to the same fault $f_{i}$. The goal is to use the best residual generator to be used in a diagnosis system. The figure shows histograms of the measurements, in the faulty case and the fault-free case, and Gaussian approximations of the data. The two residuals, $r_{1}$ and $r_{2}$, have the same pdf in the faultfree case, $q$, but different pdfs when the fault occurs, $p_{1}$ and $p_{2}$ respectively. Computing the Kullback-Leibler divergence for the both cases using (9), gives that $r_{1}$ has a higher value $\left(K\left(p_{1} \| q\right)=159.7\right)$ than $r_{2}\left(K\left(p_{2} \| q\right)=93.2\right)$.

When comparing the distributions for the two residuals in the faulty case, it is clear that the output of $r_{1}$ in the faulty case is much more separated from the fault-free case compared to $r_{2}$. Thus, $r_{1}$ gives better detectability performance compared to $r_{2}$ which also is shown by comparing the Kullback-Leibler divergences.

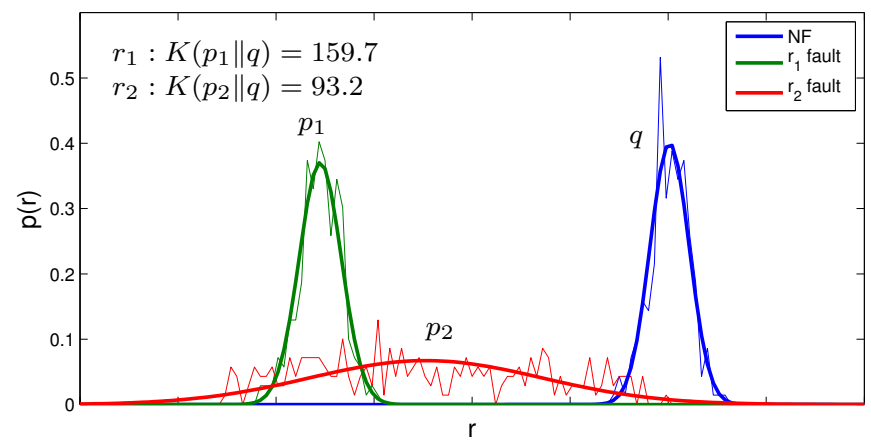

Fig. 2. Histograms and Gaussian approximations of the distributions of $r_{1}$ and $r_{2}$. The Kullback-Leibler divergence is computed based on the Gaussian approximations and shows that $r_{1}$ has a higher divergence than $r_{2}$.

The residual generator selection problem can be formulated as an optimization problem where only the residual with the highest Kullback-Leibler divergence is selected. As in the previous example shown in Fig. 2, the residual generator with the best performance $r^{*}$ for detecting a fault $f_{i}$ can be found from a set of residual generator candidates. Let $\mathcal{R}=$ $\left\{r_{1}, r_{2}, \ldots, r_{k}\right\}$ be the set of residual generator candidates 
sensitive to $f_{i}$. Then $r^{*}$ is found by solving the problem

$$
r^{*}=\arg \max _{r_{i} \in \mathcal{R}} K\left(p_{i} \| q_{i}\right),
$$

where $p_{i}$ is the distribution of $r_{i}$ in the faulty case and $q_{i}$ in the fault-free case.

Comparing the performances for all possible fault realizations, which result in different pdfs $p_{i}$, to get a general comparative measure is difficult or even impossible. Especially, for non-linear systems where detectability performance depend on the operating point. Instead, measurements or simulations from a scenario covering interesting system operating points and a representative fault realization is used as a reference. The design of such a scenario, depend on application and which fault realizations that are interesting to detect.

The Kullback-Leibler divergence is not only useful when comparing residual generator candidates. It can also be used to design new candidates which will be discussed next.

\section{METHOD}

Here, the Kullback-Leibler divergence is used to define an optimization problem to find new residual generator candidates with improved performance.

\section{A. Optimizing residual generators}

Structural methods, see e.g. [20], are used to find MSOs used to design residual generators. As described in Section III, an MSO only utilizes a minimal set of model equations to achieve analytical redundancy of order one. Thus, all possible MSOs for a given model can only generate a subset of all possible sequential residual generators, which also limits the quantitative diagnosability performance that can be achieved. By weighting residual generators based on different MSOs, thus utilizing more sensors, new residual generators with better performance could be found.

Let $\bar{r}=\left(r_{1}, r_{2}, \ldots, r_{n}\right)^{T}$, where $r_{i} \in \mathcal{R}$, be a vector of the available residual generators. Then the objective is to find weights $w \in \mathbb{R}^{n}$ such that faulty data is as separated as possible from fault-free data by solving

$$
\max _{w} K\left(p\left(w^{T} \bar{r}\right) \| q\left(w^{T} \bar{r}\right)\right)
$$

The value of (11) is invariant of the norm $\|w\|>0$, but note that (11) will be 0 when $\|w\|=0$. However, a convex search space is defined by ignoring the origo and selecting the search space as the box $-1 \leq w_{i} \leq 1, i=1, \ldots, k$.

By solving (11), the goal is to find a new single residual $r$ such that detectability of a fault mode $f_{i}$ for a given fault realization is maximized. The optimization problem (11) has a non-convex objective function which can have several local optima. Here the function fmincon in Matlab is used to apply a local interior point search method.

Example 2 (Triple-redundancy system): As a small example of numerical residual generator optimization, the tripleredundancy example in Section II is considered. Assume that three residuals have been developed to be able to detect faults in each of the three sensors given by

$$
\begin{aligned}
& r_{1}=y_{1}-y_{2} \\
& r_{2}=y_{1}-y_{3} \\
& r_{3}=y_{2}-y_{3} .
\end{aligned}
$$

Each of these three residual generators are based on one of three MSOs of the system (1), and are found by using the method presented in [20]. A fault in sensor $y_{1}$ of magnitude 1 is considered.

A Monte Carlo analysis of the optimization problem (11), where $w=\left(w_{1}, w_{2}, w_{3}\right)^{T}$ and $\bar{r}=\left(r_{1}, r_{2}, r_{3}\right)^{T}$, is made by performing the optimization for different noise realizations to analyze the robustness. The noise is approximated as i.i.d. Gaussian and 10000 samples are used here for the faulty and fault-free case. The results from the optimizations are summarized in Fig. 3 and Fig. 4, which shows that the weights $w$ and the achieved Kullback-Leibler divergence, computed by using (9), varies around the theoretical optimal value. The optimal value is computed based on the relation between the fault to noise ratio $\Gamma$ and the Kullback-Leibler divergence given by $K(p \| q)=0.5(\Gamma)^{2}$ when $p$ and $q$ have the same variances [11], and is marked in Fig. 4 by the dashed line.

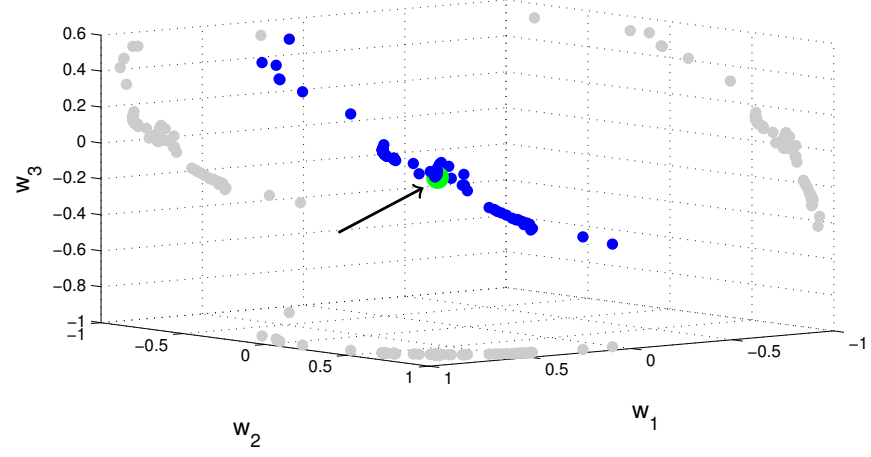

Fig. 3. Optimized parameters $w$ from different noise realizations. More than $87 \%$ of the results from the optimizations have an Euclidean distance less than 0.07 from the theoretical optimum which is marked by the arrow.

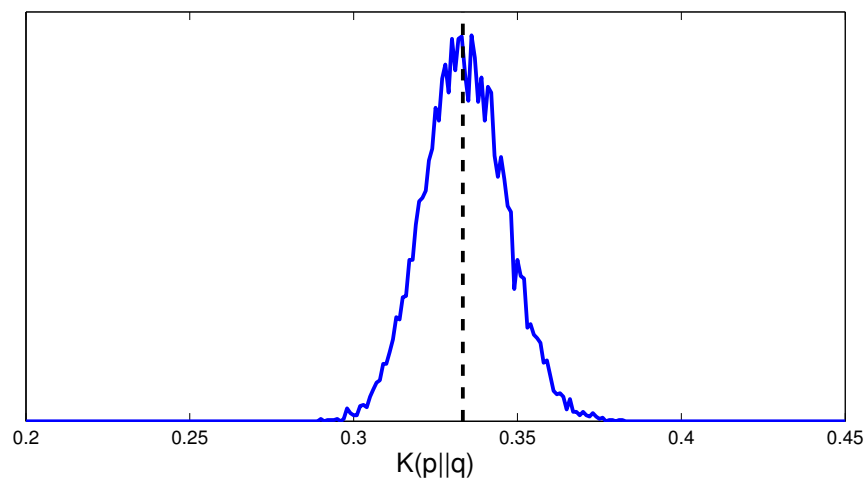

Fig. 4. The achieved Kullback-Leibler divergence when optimizing the weights $w$ for different noise realizations. The optimal value is marked by the dashed line.

In more than $87 \%$ of the cases, the optimized weights $w$ from the different realizations have an Euclidean distance 
less than 0.07 from the theoretical optimum. Also, the computed Kullback-Leibler divergence lies around the theoretical optimum. The results depends on the number of samples available to estimate the different pdfs where less samples increases the variances of the results in Fig. 3 and Fig. 4.

\section{Simulation STUdY}

Here, residuals to monitor a system of two water tanks are automatically constructed, optimized, and evaluated.

\section{A. Watertank system}

To evaluate the proposed method, a model of two serially connected watertanks as in Figure 5 is considered. The system has one known actuator signal $u$, representing the inlet flow in tank one, and four sensors $y_{1}, \ldots, y_{4}$. Sensors $y_{1}$ and $y_{2}$ measure the water levels $h_{1}, h_{2}$ and $y_{3}$ and $y_{4}$ measure the outlet flows of the two tanks. The sensor noises are modeled as i.i.d. Gaussian distributed, and the equations describing the model are given in (12). The flows are denoted $Q$, the different faults $f$, and $a$ and $b$ are parameters describing the geometry of the tanks and effective area of the pipes. The numbers in the subscripts indicate what part of the system that is considered, see Fig. 5, and some of the flows are divided into several variables using two numbers in the subscripts to be able to model several faults. The faults considered are actuation error in the pump, $f_{a}$, sensor faults, $f_{h}$ and $f_{f}$, leakage, $f_{l}$, and clogging in the pipes, $f_{c}$.

$$
\begin{array}{ll}
e_{1}: & 0=Q_{1}-u_{1}-f_{a} \\
e_{2}: & 0=Q_{2,1}-\frac{a_{1}}{b_{1}} \sqrt{h_{1}}\left(1-f_{c, 1}\right) \\
e_{3}: & 0=Q_{2,2}-Q_{2,1}\left(1-f_{l, 1}\right) \\
e_{4}: & 0=Q_{2,3}-Q_{2,2}\left(1-f_{l, 2}\right) \\
e_{5}: & 0=Q_{3,1}-\frac{a_{2}}{b_{2}} \sqrt{h_{2}}\left(1-f_{c, 2}\right) \\
e_{6}: & 0=Q_{3,2}-Q_{3,1}\left(1-f_{l, 3}\right) \\
e_{7}: & 0=\dot{h}_{1}-b_{1} Q_{1}+b_{1} Q_{2,1} \\
e_{8}: & 0=\dot{h}_{2}-b_{2} Q_{2,3}+b_{2} Q_{3,1} \\
e_{9}: & 0=y_{1}-h_{1}-f_{h, 1} \\
e_{10}: & 0=y_{2}-h_{2}-f_{h, 2} \\
e_{11}: & 0=y_{3}-Q_{2,2}-f_{f, 1} \\
e_{12}: & 0=y_{4}-Q_{3,2}-f_{f, 2}
\end{array}
$$

Simulated data from the fault-free case and the faulty modes are generated from the system given the same initial

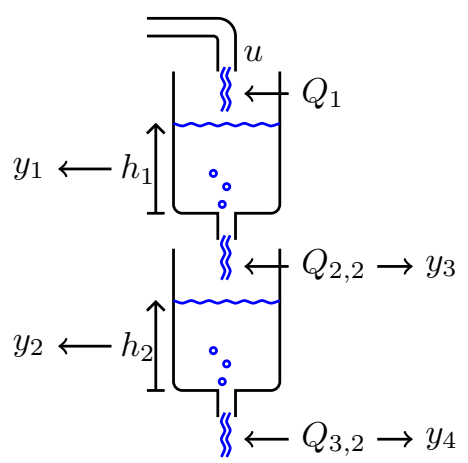

Fig. 5. The configuration of the water tank system. water levels and reference value of $h_{1}$ used in the feedback controller. Each simulation is 40 seconds and a fault is modeled as a ramped step with start at 15 seconds and maximum amplitude at 17 seconds. For quantifying the performance of each residual generator, faulty data from the period 17-40 seconds is used to calculate the distribution which is approximated as i.i.d Gaussian. The Kullback-Leibler divergence is then computed using (9) where $p$ is the pdf of data from the faulty-case and $q$ from the fault-free case.

\section{B. Results}

The structural analysis results in 17 possible MSOs where the fault sensitivity of each MSO is shown in Table I. The table is called a decision matrix and an $\mathrm{X}$ at position $(i, j)$ corresponds to that $\mathrm{MSO} i$ is sensitive to fault $j$.

TABLE I

DECISION STRUCTURE SHOWING THE FAULT SENSITIVITY OF EACH MSO DERIVED FROM (12).

\begin{tabular}{r|cccccccccc} 
& $f_{a}$ & $f_{h, 1}$ & $f_{h, 2}$ & $f_{f, 1}$ & $f_{f, 2}$ & $f_{l, 1}$ & $f_{l, 2}$ & $f_{l, 3}$ & $f_{c, 1}$ & $f_{c, 2}$ \\
\hline MSO1 & & & $\mathrm{X}$ & & $\mathrm{X}$ & & & $\mathrm{X}$ & & $\mathrm{X}$ \\
\hline MSO2 & & & $\mathrm{X}$ & $\mathrm{X}$ & $\mathrm{X}$ & & $\mathrm{X}$ & $\mathrm{X}$ & & \\
\hline MSO3 & & & $\mathrm{X}$ & $\mathrm{X}$ & & & $\mathrm{X}$ & & & $\mathrm{X}$ \\
\hline MSO4 & & & & $\mathrm{X}$ & $\mathrm{X}$ & & $\mathrm{X}$ & $\mathrm{X}$ & & $\mathrm{X}$ \\
\hline MSO5 & & $\mathrm{X}$ & & $\mathrm{X}$ & & $\mathrm{X}$ & & & $\mathrm{X}$ & \\
\hline MSO6 & & $\mathrm{X}$ & $\mathrm{X}$ & & $\mathrm{X}$ & $\mathrm{X}$ & $\mathrm{X}$ & $\mathrm{X}$ & $\mathrm{X}$ & \\
\hline MSO7 & & $\mathrm{X}$ & $\mathrm{X}$ & & & $\mathrm{X}$ & $\mathrm{X}$ & & $\mathrm{X}$ & $\mathrm{X}$ \\
\hline MSO8 & & $\mathrm{X}$ & & & $\mathrm{X}$ & $\mathrm{X}$ & $\mathrm{X}$ & $\mathrm{X}$ & $\mathrm{X}$ & $\mathrm{X}$ \\
\hline MSO9 & $\mathrm{X}$ & $\mathrm{X}$ & & $\mathrm{X}$ & & $\mathrm{X}$ & & & & \\
\hline MSO10 & $\mathrm{X}$ & $\mathrm{X}$ & $\mathrm{X}$ & & $\mathrm{X}$ & $\mathrm{X}$ & $\mathrm{X}$ & $\mathrm{X}$ & & \\
\hline MSO11 & $\mathrm{X}$ & $\mathrm{X}$ & $\mathrm{X}$ & & & $\mathrm{X}$ & $\mathrm{X}$ & & & $\mathrm{X}$ \\
\hline MSO12 & $\mathrm{X}$ & $\mathrm{X}$ & & & $\mathrm{X}$ & $\mathrm{X}$ & $\mathrm{X}$ & $\mathrm{X}$ & & $\mathrm{X}$ \\
\hline MSO13 & $\mathrm{X}$ & $\mathrm{X}$ & & & & & & & $\mathrm{X}$ & \\
\hline MSO14 & $\mathrm{X}$ & & & $\mathrm{X}$ & & $\mathrm{X}$ & & & $\mathrm{X}$ & \\
\hline MSO15 & $\mathrm{X}$ & & $\mathrm{X}$ & & $\mathrm{X}$ & $\mathrm{X}$ & $\mathrm{X}$ & $\mathrm{X}$ & $\mathrm{X}$ & \\
\hline MSO16 & $\mathrm{X}$ & & $\mathrm{X}$ & & & $\mathrm{X}$ & $\mathrm{X}$ & & $\mathrm{X}$ & $\mathrm{X}$ \\
\hline MSO17 & $\mathrm{X}$ & & & & $\mathrm{X}$ & $\mathrm{X}$ & $\mathrm{X}$ & $\mathrm{X}$ & $\mathrm{X}$ & $\mathrm{X}$ \\
\hline & & & & & & & & & &
\end{tabular}

The table shows that there are several MSOs that can be used to detect each fault. However, the information in Table I does not include which MSO that is the best at detecting each fault, nor which sequential residual generator based on an MSO that performs best. In Fig. 6, the fault $f_{h, 1}$ is induced at 15 seconds and the eight residuals based on MSO6, which are sensitive to the fault, are plotted. The performance of the residuals are different from each other where one residual is clearly better than the other seven. The result shows that the design of the residual generator based on the MSO clearly affects the achieved performance.

By using the previous approach described in Section 4 for all MSOs, the best residual generator for each MSO can be selected for each fault, and the single best residual generator can be found by comparing the different solutions. However, the MSOs are based on a minimal set of sensors, thus only a limited amount of information is used to detect a fault. New residual generators can be optimized to improve the detectability performance as described in Section V. Here, detection of a sensor fault $f_{h, 1}$ is considered where the best residual generators from each of the 17 MSOs are used in the optimization. 


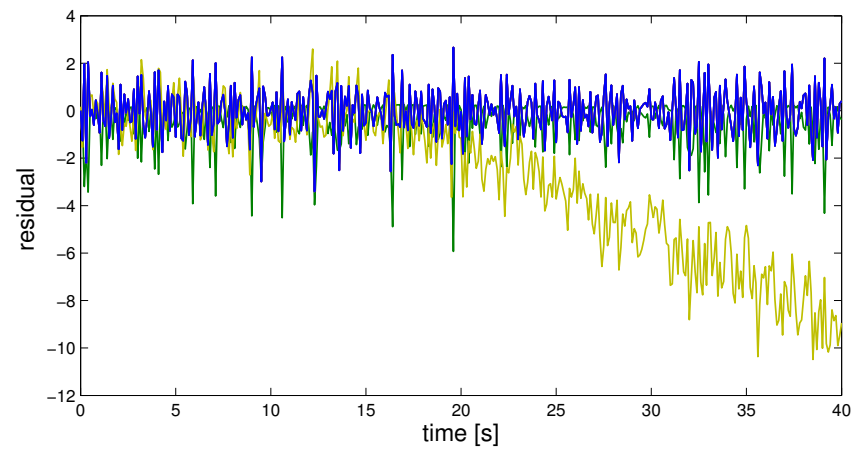

Fig. 6. The residual generators based on MSO6 when $f_{h, 1}$ has occurred at $t=15 \mathrm{~s}$. There are eight residual generators, where one clearly deviates from zero.

To visualize the achieved performance of the optimization, Fig. 7 compares two residuals. One residual is the best residual possible to design based on any MSO, and the other residual is optimized using (11). Both tests are normalized to have the same noise variance in the fault-free case. The results show that the optimized residual generator achieves better performance compared to the single best sequential residual generator.

In Fig. 6, the single best residual generator based on MSO6 looks like a ramp. However, when comparing the best residual generators for each MSO, the residual generator based on MSO13 is the best because of higher KullbackLeibler divergence. This residual is shown in Fig. 7 and responds as a step to the fault. When weighting the residual generators together using (11), the fault to noise ratio $\Gamma$ is increased and the new optimized residual generator is better than the residual generator based on only one MSO, and behaves like a ramp when the fault occurs.

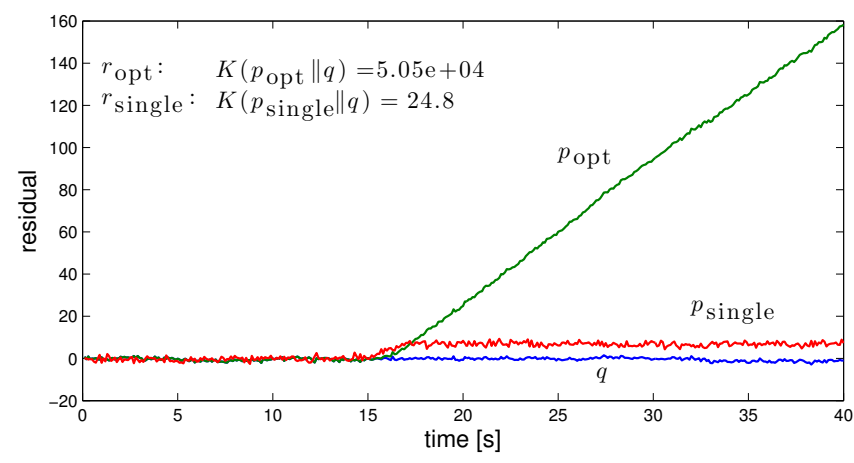

Fig. 7. The residuals when $f_{h, 1}$ has occurred at $t=15 \mathrm{~s}$.

\section{CONCLUSIONS}

A structural method for generating residual generators based on MSOs is improved by using the Kullback-Leibler divergence to quantify the diagnosability performance. Thus, different residual generators can be compared and the best one can be automatically selected. An example of triple redundancy is used to show that performance can be improved by weighting all available sensors in an optimal way instead of only doing pair-wise comparison.

Also, by weighting several residual generators together using an optimization algorithm, new residual generators with better performance can be found. The proposed method is evaluated on a model of a watertank system. Results show that the achieved performance can be significantly improved by taking quantitative diagnosability performance into consideration when selecting the residual generator. The proposed method can be used for improving performance of automatically designed diagnosis systems.

\section{REFERENCES}

[1] M. Blanke, M. Kinnaert, J. Lunze, and M. Staroswiecki, Diagnosis and Fault-Tolerant Control, 2nd ed. Springer, 2006.

[2] J. Armengol, A. Bregon, T. Escobet, E. R. Gelso, M. Krysander, M. Nyberg, X. Olive, B. Pulido, and L. Trave-Massuyes, "Minimal structurally overdetermined sets for residual generation: A comparison of alternative approaches," in Proceedings of IFAC Safeprocess'09, Barcelona, Spain, 2009.

[3] C. Sundström, E. Frisk, and a. L. Nielsen, "Selecting and utilizing sequential residual generators in FDI applied to hybrid vehicles," IEEE Trans. on SMC: Systems, Accepted for publication.

[4] C. Svärd and M. Nyberg, "Residual generators for fault diagnosis using computation sequences with mixed causality applied to automotive systems," IEEE Trans. on SMC - Part A, vol. 40, no. 6, pp. 1310-1328, 2010.

[5] M. Nyberg, "Criterions for detectability and strong detectability of faults in linear systems," International Journal of Control, vol. 75, no. 7, pp. 490-501, May 2002.

[6] C. Chen and R. Patton, Robust Model-Based Fault Diagnosis For Dynamic Systems, ser. Kluwer International Series on Asian Studies in Computer and Information Science, 3. Kluwer, 1999.

[7] T. J. Wheeler, "Probabilistic Performance Analysis of Fault Diagnosis Schemes," Ph.D. dissertation, University of California, Berkeley, 2011.

[8] S. M. Kay, Fundamentals of statistical signal processing: Detection theory. Upper Saddle River, NJ, USA: Prentice-Hall, Inc., 1998.

[9] G. Casella and R. L. Berger, Statistical Inference. Pacific Grove, CA: Duxbury Resource Center, 2001.

[10] M. Basseville, "On fault detectability and isolability," European Journal of Control, vol. 7, no. 6, pp. 625 - 637, 2001.

[11] D. Eriksson, E. Frisk, and M. Krysander, "A method for quantitative fault diagnosability analysis of stochastic linear descriptor models," Automatica, vol. 49, no. 6, pp. 1591 - 1600, 2013.

[12] D. Eriksson, "Diagnosability analysis and FDI system design for uncertain systems," Tech. Rep., 2013, liU-TEK-LIC-2013:18, Thesis No. 1584.

[13] J. Li, W. Yang, Y. Zhang, Y. Pei, Y. Ren, and W. Wang, "Aircraft vulnerability modeling and computation methods based on product structure and \{CATIA\}," Chinese Journal of Aeronautics, vol. 26, no. 2 , pp. $334-342,2013$.

[14] R. Isermann, Fault Diagnosis Systems - An Introduction from fault Detection to Fault Tolerance. Springer Verlag, 2006.

[15] P. M. Frank, "Enhancement of robustness in observer-based fault detection," Int. J. of Control, vol. 59, no. 4, pp. 955-981, 1994.

[16] M. Staroswiecki and P. Declerck, "Analytical redundancy in non-linear interconnected systems by means of structural analysis," in Proceedings of IFAC AIPAC'89, Nancy, France, 1989, pp. 51-55.

[17] M. Staroswiecki and G. Comtet-Varga, "Analytical redundancy relations for fault detection and isolation in algebraic dynamic systems," Automatica, vol. 37, no. 5, pp. 687 - 699, 2001.

[18] M. Krysander, J. Åslund, and M. Nyberg, "An efficient algorithm for finding minimal over-constrained sub-systems for model-based diagnosis," IEEE Trans. on SMC - Part A: Systems and Humans, vol. 38 , no. $1,2008$.

[19] L. Barford, E. Manders, G. Biswas, P. Mosterman, V. Ram, and J. Barnett, "Derivative estimation for diagnosis," HP Laboratories Palo Alto, Tech. Rep., 1999, HPL-1999-18.

[20] M. Krysander, "Design and analysis of diagnosis systems using structural methods," Ph.D. dissertation, Linköpings universitet, June 2006. 